\title{
Fluid Balance of the Second Day Following Operation is Associated with Early Mortality and Multiorgan Failure After Pericardiectomy for Constrictive Pericarditis
}

\author{
Jing-bin Huang, MD, Zhao-ke Wen, MD, Wei-jun Lu, MD, Chang-chao Lu, MD, Xian-ming Tang, MD, \\ Xiang-wei Li, MD, Xin Deng, MD \\ Department of Cardiothoracic Surgery, The People’s Hospital of Guangxi Zhuang, Nanning, Guangxi, China
}

\section{ABSTRACT}

Background: The operative mortality of pericardiectomy still is high. This retrospective study was conducted to determine the risk factors of early mortality and multiorgan failure.

Methods: We retrospectively analyzed patients undergoing pericardiectomy from January 2009 to June 2020 at our hospital. Pericardiectomy was performed via sternotomy. Histopathologic studies of pericardium tissue from every patient were done. All survivors were monitored to the end date of the study.

Results: Ninety-two consecutive patients undergoing pericardiectomy for constrictive pericarditis were included in the study. Postoperatively, central venous pressure significantly decreased, and left ventricular end diastolic dimension and left ventricular ejection fractions significantly improved. The overall mortality rate was $5.4 \%$. The common postoperative complications include acute renal injury (27.2\%), and multiorgan failure (8.7\%). Analyses of risk factors showed that fluid balance of the second day following operation is associated with early mortality and multiorgan failure. In this series from Guangxi, China, characteristic histopathologic features of tuberculosis $(60 / 92,65.2 \%)$ of pericardium were the most common histopathologic findings, and 32 patients $(32 / 92,34.8 \%)$ had the histopathologic findings of chronic nonspecific inflammatory changes. The functional status of the patients improved after pericardiectomy; 6 months later postoperatively 85 survivors were in class I $(85 / 87,97.7 \%)$ and two were in class II $(2 / 87,2.3 \%)$.

Conclusions: Tuberculosis is the most common cause of constrictive pericarditis in Guangxi, China. Fluid balance of the second day following operation is associated with early mortality and multiorgan failure after pericardiectomy for constrictive pericarditis in our study.

Received April 30, 2021; accepted May 27, 2021.

Correspondence: Fing-bin Huang, MD, Department of Cardiothoracic Surgery, The People's Hospital of Guangxi Zhuang Autonomous Region, 6 Taoyuan Road, Nanning, 530021, Guangxi, China, Telephone 86-771-2188205, Fax 86-771-2188214 (e-mail: bjb010222@163.com).

\section{INTRODUCTION}

Constrictive pericarditis is a progressive and disabling disease. It arises as a result of the fibrous thickening and calcification of the pericardium due to chronic inflammatory changes from various injuries that impair diastolic filling, reducing cardiac output and ultimately leading to left and right heart failure. Patients with constrictive pericarditis undergo pericardiectomy with symptomatic improvement in more than $90 \%$ of them after the procedure [Acharya 2018; Depboylu 2017; Melo 2019]. The operative mortality risk of pericardiectomy still is high, ranging between $5 \%$ and $20 \%$ [Mori 2019; Calderon-Rojas 2020].

This retrospective study was conducted to determine the risk factors of early mortality and multiorgan failure (MOF).

\section{PATIENTS AND METHODS}

Design: This was a retrospective, observational cohort study of patients who underwent pericardiectomy from January 2009 to June 2020 at The People's Hospital of Guangxi Zhuang Autonomous Region. Medical records were reviewed to collect the data of the variables to be analyzed. Eligibility and inclusion: Patients who underwent pericardiectomy between from January 2009 to June 2020 at our hospital. Excluded were patients with incomplete or missing medical records.

Variables to be analyzed: Gender (female/male), age, weight before diuresis, weight after diuresis, NYHA class, cachexia, pulmonary tuberculosis, rheumatic heart disease, infective endocarditis, valvular heart disease, coronary heart disease, pleural effusion, left ventricular end diastolic dimension, left ventricular ejection fractions, aortic insufficiency, mitral regurgitation, tricuspid regurgitation, thickened pericardium, pericardial effusion, pericardial calcification, serum creatinine, mean intubation time, ICU retention time, hospitalization time after surgery, central venous pressure, postoperative chest drainage, surgical duration, fresh-frozen plasma, packed red cells, fluid balance on operation day, the first day following operation and the second day following operation, low cardiac output syndrome, acute renal injury, multiorgan failure, longterm intubation, empyema, hepatic failure, respiratory failure, ventricular fibrillation, use of inotropic medication, extracorporeal membrane oxygenation (ECMO) requirement, and death. 
Low cardiac output syndrome: All patients were monitored with a pulmonary artery catheter in the operation room and intensive care unit. Cardiac output (CO) and venous oxygen saturation of hemoglobin continuously were measured. Low cardiac output syndrome is defined by a cardiac index (CI) of less than $2.0 \mathrm{~L} / \mathrm{min} / \mathrm{m} 2$ in the operation room and intensive care unit [Epting 2016; Chandler 2016].

Preoperative diuresis protocol: Hydrochlorothiazide tablet $25 \mathrm{mg}$ bid, furosemide tablet $20 \mathrm{mg}$ bid. Diuresis treatment last seven to 30 days. Postoperative left ventricular enddiastolic diameter (LVEDD) was measured by transthoracic echocardiography postoperatively one to seven days in the intensive care unit. Perioperative death was defined as death within 30 days of the operation or during the same hospital admission.

In our study, serum creatinine was used as the diagnostic standard of acute renal injury. According to Kidney Disease Improving Global Outcomes (KDIGO) classification, if serum creatinine increases by $\geq 0.3 \mathrm{mg} / \mathrm{dl}(26.5 \mu \mathrm{mol} / \mathrm{l})$ within 48 hours, serum creatinine is $50 \%$ higher than the baseline within the first seven days, or urine output is below $0.5 \mathrm{ml} /$ $\mathrm{kg} /$ hour for six hours, the patient is considered to have acute renal injury [Howitt 2018; Jacob 2019].

Multiorgan failure (MOF) is regarded as a continuous process of varying levels of organ failure rather than an all-ornone event [Epting 2016]. To characterize MOF, six different organ systems are regarded as "key organs:" lungs, cardiovascular system, kidneys, liver, coagulation system, and central nervous system [Durham 2003].

Hepatic failure is defined as "a severe liver injury, potentially reversible in nature and with onset of hepatic encephalopathy within eight weeks of the first symptoms in the absence of pre-existing liver disease" [Bernal 2013].

Respiratory failure is a condition in which the respiratory system fails in one or both of its gas exchange functions, i.e. oxygenation of and/or elimination of carbon dioxide from mixed venous blood. It is defined by an arterial oxygen tension $(\mathrm{Pa}, \mathrm{O} 2)$ of $\leq 8.0 \mathrm{kPa}(60 \mathrm{mmHg})$, an arterial carbon dioxide tension ( $\mathrm{Pa}, \mathrm{CO} 2)$ of $\geq 6.0 \mathrm{kPa}(45 \mathrm{mmHg})$, or both [Roussos 2003; Nashef 2012].

Statistical analyses: Continuous variables are reported as means \pm SE. The chi-square test, Kruskal-Walls test or Wilcoxon rank-sum test, as appropriate, were used to evaluate relationships between the preoperative variables, and selected intraoperative and postoperative variables. The relationships with perioperative risk factors were assessed by means of contingency table methods and logistic regression analysis. $P$ values less than 0.05 were considered to be statistically significant. All analyses were performed using IBM SPSS version 24.0 software (IBM SPSS Inc., USA).

Ethics approval: The experiment protocol for involving humans was in accordance with the Helsinki Statement and national guidelines and approved by the Medical Ethics Committee of The People's Hospital of Guangxi Zhuang Autonomous Region. The Medical Ethics Committee of The People's Hospital of Guangxi Zhuang Autonomous Region gave the authors approval to waive the need for patient consent for publishing data in the study about the patients.

\section{RESULTS}

Characteristics of the population under study: Ninetytwo consecutive patients undergoing pericardiectomy for constrictive pericarditis were included in the study. (Table 1) No patients met the exclusion criteria. Forty-four patients (44/92, 47.8\%) had constrictive pericarditis associated with pericardial effusion. Cardiopulmonary bypass was performed in eight patients $(8 / 92,8.7 \%)$ with concomitant valve replacement. Only two patients underwent cardiac catheterization.

Diagnosis of constrictive pericarditis: The diagnosis of constrictive pericarditis was made on the basis of clinical manifestation, echocardiography, chest computed tomography (CT) scan, cardiac catheterization, surgery, and pathological criteria. The most important diagnostic tool is the suspicion of constrictive pericarditis in a patient with signs and symptoms of right-sided heart failure that are disproportionate to pulmonary of left-sided heart disease. Typical symptoms and signs are a prominent change in the $\mathrm{x}$ and $\mathrm{y}$ descent in jugular venous pulse, dyspnea upon exertion, palpitations, abdominal distension, as well as edema in the ankles or legs.

Table 1. Preoperative characteristics of patients $(N=92)$

\begin{tabular}{|c|c|}
\hline Variable & Value \\
\hline Female/male (n) & $59 / 33$ \\
\hline Age (years) & $52.83 \pm 1.56$ (range 17.0 to 74.0 ) \\
\hline Weight before diuresis $(\mathrm{kg})$ & $55.97 \pm 0.94$ (range 36.0 to 81.0 ) \\
\hline Weight after diuresis (kg) & $53.0 \pm 0.74$ (range 34.0 to 72.0 ) \\
\hline \multicolumn{2}{|l|}{ NYHA class } \\
\hline II & $53(57.6 \%)$ \\
\hline III & $38(41.3 \%)$ \\
\hline IV & $1(1.1 \%)$ \\
\hline Cachexia (n) & $3(3.3 \%)$ \\
\hline Pulmonary tuberculosis ( $n$ ) & $2(2.2 \%)$ \\
\hline Rheumatic heart disease (n) & $3(3.3 \%)$ \\
\hline Infective endocarditis (n) & $1(1.1 \%)$ \\
\hline Valvular heart disease $(n)$ & $3(3.3 \%)$ \\
\hline Coronary heart disease (n) & $3(3.3 \%)$ \\
\hline Pleural effusion (n) & $8(8.7 \%)$ \\
\hline Preoperative LVEDD (mm) & $40.82 \pm 0.36$ (range 29.0 to 49.0 ) \\
\hline Preoperative LVEF (mm) & $0.62 \pm 0.01$ (range 0.51 to 0.74 ) \\
\hline Aortic insufficiency (n) & $6(6.5 \%)$ \\
\hline Mitral regurgitation (n) & $8(8.7 \%)$ \\
\hline Tricuspid regurgitation (n) & $5(5.4 \%)$ \\
\hline Thickened pericardium (n) & $91(98.9 \%)$ \\
\hline Pericardial effusion (n) & $44(47.8 \%)$ \\
\hline Pericardial calcification (n) & $15(16.3 \%)$ \\
\hline Baseline serum creatinine $(\mu \mathrm{mol} / \mathrm{l})$ & $80.6 \pm 3.6$ (range 45.0 to 284.0 ) \\
\hline
\end{tabular}


Echocardiography and chest computed tomography (CT) scan revealed a severely thickened or calcified pericardium, and cardiac catheterization revealed elevated end-diastolic pressure and the "square root sign" of right ventricular pressure tracing. Surgical and pathological findings were reviewed to confirm the preoperative diagnosis [Gatti 2020; Wei 2019; Armstrong 2020; Vondran 2019]. (Figure 1) (Figure 2)

Surgical results: Pericardiectomy was performed via sternotomy. The pericardium was removed between the two phrenic nerves and from the great vessels to the basal aspect of the heart. Constricting layers of epicardium were removed when it is possible. The pericardium was palpated to identify

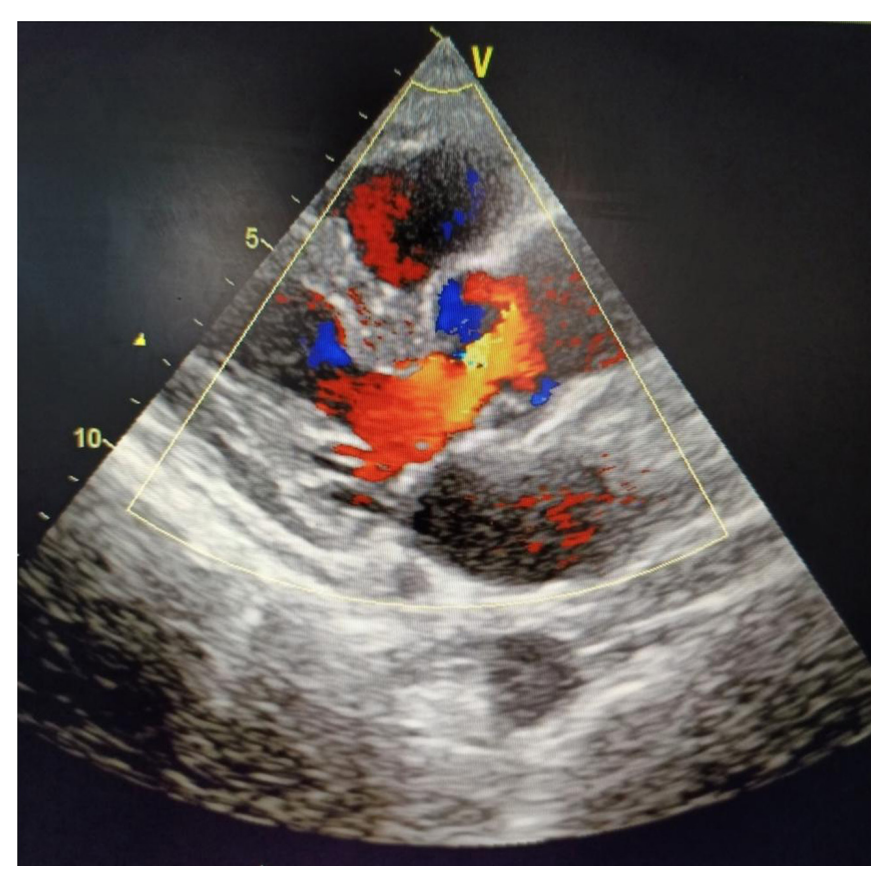

Figure 1. Transthoracic echocardiography shows the thickened pericardium.

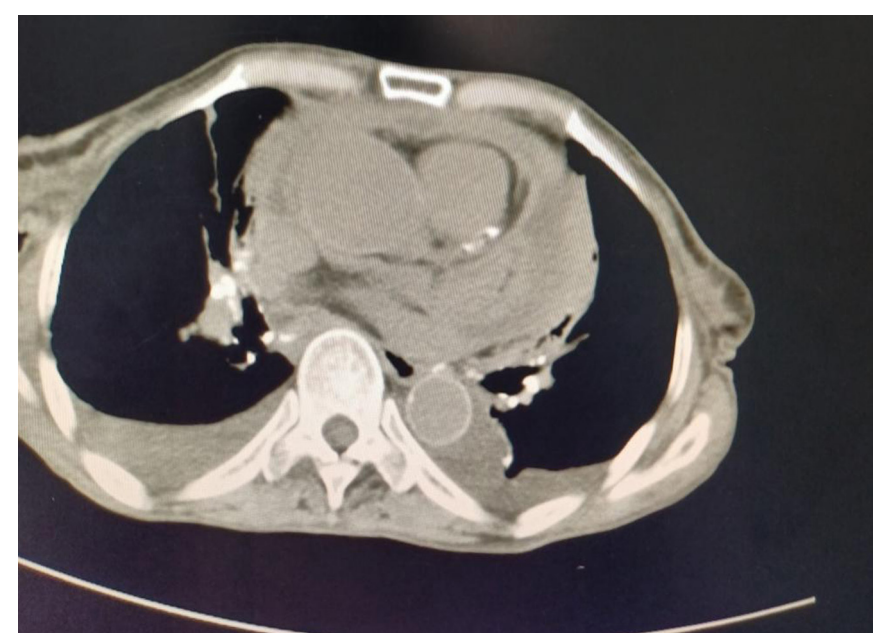

Figure 2. Chest computed tomographic scan shows the significantly thickened pericardium. a relatively soft and uncalcified area after median sternotomy, and the thymus laterally was removed. An \#-shaped incision was made over the pericardium. Dissection was started at the base of the aorta, extended downward to the lateral and posterior walls of the left ventricle, and followed by the diaphragmatic pericardium. The pericardium over the right atrium and venae cavae was resected last. The myocardium was then exposed to achieve mobilization of the heart down to the phrenic nerves. If calcified plaque penetrating the epicardium was present, we left small "islands" of calcified pericardial tissue. Cardiopulmonary bypass (CPB) was avoided during surgery except for concomitant valve replacement. (Table 2)

Postoperatively, CVP decreased statistically significantly $(P<0.001)$, and LVEDD and LVEF improved statistically significantly $(P<0.001$ and $P<0.001$, respectively). (Table 3 )

The overall mortality rate in this series was $5.4 \%(5 / 92)$, and the five patients died of multiorgan failure. All patients with multiple organ failure $(N=5)$ had acute renal injury, which accounted for $20 \%(5 / 25)$ of patients with acute renal injury (Table 2 and Table 4).

The common early postoperative complications included acute renal injury $(25 / 92,27.2 \%$, and multiorgan failure $(8 / 92,8.7 \%)$. (Table 4)

Table 5 showed the use of inotropic medication $(\mathrm{N}=92)$. (Table 5)

D0 fluid balance $=$ fluid balance on operation day $=$ inputoutput; D1 fluid balance $=$ fluid balance of the first day

Table 2. Operative data $(N=92)$

\begin{tabular}{|c|c|}
\hline Variable & Value \\
\hline Mean intubation time (days) & $75.89 \pm 8.69$ (range 3.0 to 336.0 ) \\
\hline ICU retention time (days) & $5.32 \pm 0.53$ (range 1.0 to 32.0 ) \\
\hline Hospitalized time after surgery (days) & $16.05 \pm 1.16$ (range 1.0 to 99.0 ) \\
\hline Postoperative CVP (mmHg) & $11.62 \pm 0.31$ (range 5 to 20 ) \\
\hline D0 fluid balance $(\mathrm{ml})$ & $-1269.5 \pm 87.5$ (range -3100 to 500$)$ \\
\hline D1 fluid balance (ml) & $-777.5 \pm 124.8$ (range -4320 to 1830$)$ \\
\hline D2 fluid balance $(\mathrm{ml})$ & $-777.6 \pm 73.1$ (range -2700 to 700$)$ \\
\hline Postoperative chest drainage $(\mathrm{ml})$ & $932.9 \pm 57.4$ (range 150 to 2300 ) \\
\hline Postoperative LVEDD (mm) & $42.7 \pm 0.3$ (range 36.0 to 49.0 ) \\
\hline Postoperative LVEF (mm) & $0.66 \pm 0.01$ (range 0.52 to 0.79 ) \\
\hline Surgical duration (min) & $190.5 \pm 6.6$ (range 80.0 to 390.0$)$ \\
\hline Fresh-frozen plasma (ml) & $929.9 \pm 95.2$ (range 0.0 to 5250.0 ) \\
\hline Packed red cells (unit) & $0.42 \pm 0.10$ (range 0.0 to 4.0$)$ \\
\hline
\end{tabular}

Serum creatinine $24 \mathrm{~h}$ after surgery $(\mu \mathrm{mol} / \mathrm{I})$

$81.6 \pm 2.8$ (range 23.0 to 178.0 )

Serum creatinine $48 \mathrm{~h}$ after surgery $(\mu \mathrm{mol} / \mathrm{l})$

Amount of fluid on DO $(\mathrm{ml})$

$96.7 \pm 4.5$ (range 39.0 to 226.0 )

Amount of fluid on D1 $(\mathrm{ml})$

Amount of fluid on D2 (ml)
$1856.3 \pm 58.9$ (range 526.0 to 3123.0 )

$2621.0 \pm 63.4$ (range 1174.0 to 4481.0)

$2512.9 \pm 47.8$ (range 1192.0 to 3665.0 ) 
Table 3. Operative results

\begin{tabular}{lccc}
\hline Clinical data & Preoperative & Postoperative & $P$-value \\
\hline $\operatorname{CVP}(\mathrm{mmHg})(\mathrm{N}=92)$ & $20.42 \pm 0.50$ & $11.62 \pm 0.31$ & $<0.001$ \\
$\operatorname{LVEDD}(\mathrm{mm})(\mathrm{N}=88)$ & $40.82 \pm 0.37$ & $42.73 \pm 0.34$ & $<0.001$ \\
$\operatorname{LVEF}(\%)(\mathrm{N}=88)$ & $62 \pm 1$ & $66 \pm 1$ & $<0.001$ \\
\hline
\end{tabular}

CVP, central venous pressure; LVEDD, left ventricular end diastolic dimension; LVEF, left ventricular ejection fraction

Table 4. Postoperative complications $(N=92)$

\begin{tabular}{lc}
\hline Complication & $N(\%)$ \\
\hline Acute renal injury & $25(27.2 \%)$ \\
Multiorgan failure & $8(8.7 \%)$ \\
Long-term intubation $>48 \mathrm{~h}$ & $44(47.8 \%)$ \\
Empyema & $1(1.1 \%)$ \\
Hepatic failure & $1(1.1 \%)$ \\
Respiratory failure & $3(3.3 \%)$ \\
Ventricular fibrillation & $2(2.2 \%)$
\end{tabular}

Table 5. Use of inotropic medication $(N=92)$

\begin{tabular}{lc}
\hline Inotropic medication & $N(\%)$ \\
\hline Dopamine & $5(56.5 \%)$ \\
Milrinone & $2(2.2 \%)$ \\
Dopamine+milrinone & $4(4.3 \%)$ \\
Dopamine+adrenaline & $1(15.2 \%)$ \\
Dopamine+adrenaline+milrinone & $1(13.0 \%)$ \\
Dopamine+adrenaline+milrinone+norepinephrine & $2(2.2 \%)$ \\
Dopamine+norepinephrine+milrinone+levosimendan & $2(2.2 \%)$ \\
Dopamine+adrenaline+norepinephrine & $2(2.2 \%)$
\end{tabular}

following operation; D2 fluid balance $=$ fluid balance of the second day following operation; $\mathrm{D} 0=$ operation day; $\mathrm{D} 1=$ the first day following operation; D2 = the second day following operation; the second day.

Histopathologic study results: Histopathologic studies of pericardium tissue from every patient was done. The diagnosis of tuberculosis was confirmed on the basis of clinical findings and histopathologic features, including the presence of typical granuloma and caseous necrosis, acidfast bacilli in Ziel-Nelson tissue staining, and bacteriologic studies using the polymerase chain reaction (PCR) test on the pericardial fluid or tissue for evidence of mycobacterium tuberculosis.

In this series from Guangxi, China, characteristic histopathologic features of tuberculosis $(60 / 92,65.2 \%)$ of pericardium were the most common histopathologic findings, and 32 patients $(32 / 92,34.8 \%)$ had the histopathologic findings of chronic nonspecific inflammatory changes. (Figure 3) (Figure 4) Table 7 shows the comparison of patients with tubercular and nonspecific inflammatory pericarditis in the series. (Table 7)

Follow-up results: All survivors discharged from hospital were monitored to the end date of the study. In the outpatient department, all patients were investigated with echocardiogram, electrocardiogram, and X-ray chest film once every 3 to 12 months. At the last follow up, the patients were contacted by telephone or micromassage or interviewed directly in the outpatient department.

The functional status of the patients improved after pericardiectomy. Fifty-three of the 92 patients $(57.6 \%)$ had been in NYHA class II, 38 of $92(41.3 \%)$ in class III, and one of $92(1.1 \%)$ in class IV before the operation, but 6 months later postoperatively 85 survivors were in class I $(85 / 87,97.7 \%)$ and two were in class II $(2 / 87,2.3 \%)$.

Analysis of risk factors of early mortality: Univariate analysis of potential risk factors of early mortality showed that several factors were associated with early mortality, including D1 fluid balance $(P=0.038)$, D2 fluid balance $(P=0.015)$, weight after diuresis $(P=0.020)$, surgical duration $(P=0.048)$, postoperative CVP $(P=0.042)$, and age $(P=0.014)$. When they were included in multivariate analysis models, multivariate analyses also showed that D1 fluid balance $(P=0.034), \mathrm{D} 2$ fluid balance $(P=0.017)$, and age $(P=0.024)$ are associated with early mortality. (Table 6)

Analysis of risk factors of multiorgan failure: Univariate analysis of potential risk factors of multiorgan failure showed that several factors were associated with multiorgan failure, including D2 fluid balance $(P=0.002)$, postoperative chest drainage $(P=0.042)$, weight before diuresis $(P=0.032)$, surgical duration $(P=0.003)$, intubation time $(P<0.001)$, ICU retention time $(P<0.001)$, preoperative CVP $(P=0.001)$, preoperative LVEDD $(P=0.047)$, fresh-frozen plasma $(P=0.001)$, and packed red cells $(P=0.002)$. When they were included in multivariate analysis models, multivariate analyses also showed that D2 fluid balance $(P=0.042)$, surgical duration $(P=0.011)$, preoperative CVP $(P=0.005)$, and ICU retention time $(P=$ 0.025 ) are associated with multiorgan failure (Table 6).

Fluid balance of the second day following operation is associated with early mortality $(P=0.017)$ and multiorgan failure $(P=0.042)$ after pericardiectomy for constrictive pericarditis in our study (Table 6).

\section{DISCUSSION}

Constrictive pericarditis is defined as the chronic fibrous thickening of the wall of the pericardial sac, resulting in abnormal diastolic filling. Surgical pericardiectomy is extremely effective and possibly restorative for the heart failure, while it is particularly challenging because of the increased risk of right heart failure [Fang 2020; Ahmad 2019].

Surgical removal of the pericardium is associated with an operative mortality rate of $5 \%$ to $20 \%$ in various large series. Myocardial atrophy after prolonged constriction, residual 


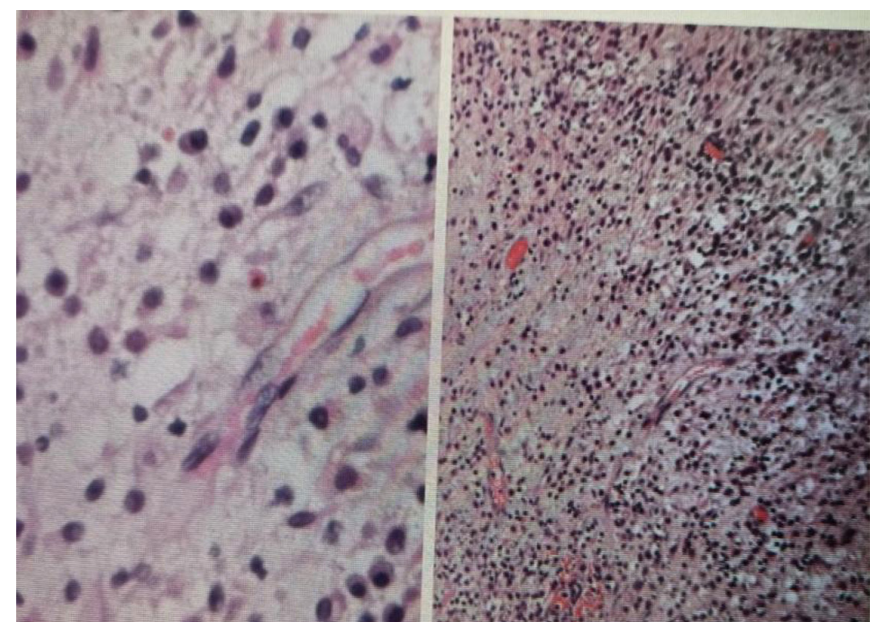

Figure 3. Histopathologic studies of pericardium tissue from patients show the characteristic histopathologic features of tuberculosis including typical granuloma and caseous necrosis.

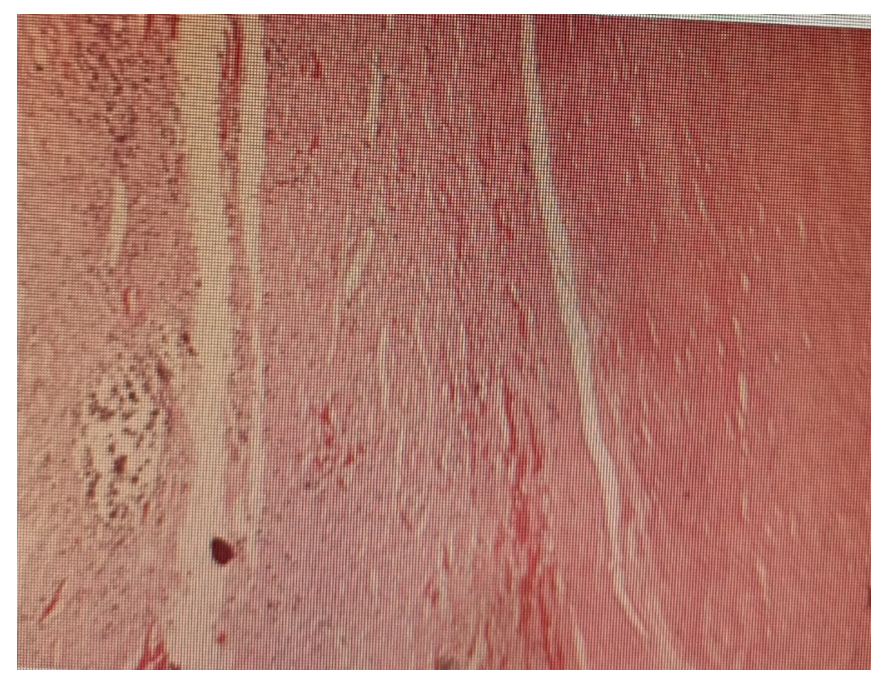

Figure 4. Histopathologic studies of pericardium tissue from patients show the histopathologic findings of chronic nonspecific inflammatory changes.

constriction, or a concomitant myocardial process can lead to prolonged cardiac failure despite successful pericardiectomy [Rupprecht 2018; Chang 2019].

Surgical technique: In order to avoid pulmonary edema and heart damage due to excessive expansion because a large amount of tissue fluid flows back the heart and lungs, the pericardium was decorticated in the following order: left ventricular outflow tract - apex of heart - right ventricular outflow tract - right ventricle - superior and inferior vena cava entrance - pericardium diaphragm surface.

If the pericardium calcification is serious and closely adheres to the myocardium, it is difficult to completely peel off the pericardium. The local \#-shaped incision is used to release the pericardium [Welch 2017; Welch 2015].

Fluid management in these patients undergoing pericardiectomy: Fluid balance of the second day following

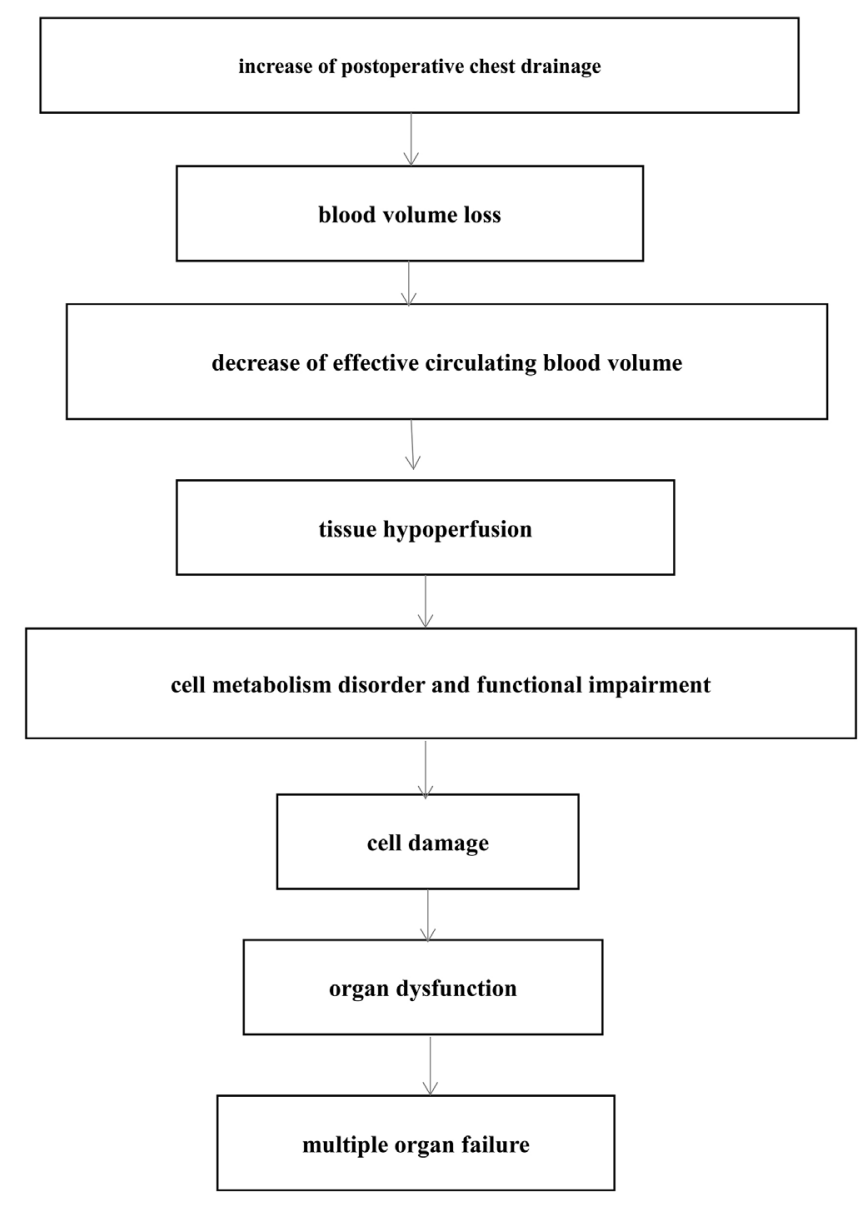

Figure 5. Postoperative chest drainage can affect the incidence of multiorgan failure.

operation is associated with early mortality and multiorgan failure after pericardiectomy for constrictive pericarditis in our study (Table 6).

Perfect preoperative preparation is very important for reducing postoperative complications and smooth recovery. The recovery of patients postoperatively depends on the right timing of operation, extent of pericardial dissection, and perioperative management [Welch 2018; Miranda 2017; Hemmati 2017].

During the process of pericardial dissection, acute low cardiac output syndrome may occur due to acute cardiac dilatation, especially after pericardium was removed from the surface of the right ventricle. Because of systemic venous hypertension, the ventricle rapidly fills and expands, resulting in acute low cardiac output syndrome. Therefore, fluid input should be limited during the operation. Inotropes and furosemide should be applied immediately after the left ventricular constriction is relieved. At the same time, too much fluid should be avoided to relieve the heart burden. Dopamine and other catecholamines should be used. If the drug response is poor and the low cardiac output cannot be corrected, ECMO should be used [Unai 2019; Yunfei 2019].

The volume and speed of fluid input should be strictly controlled to avoid sudden increase of heart burden to cause 
Table 6. Analysis of risk factors of early mortality and multiorgan failure

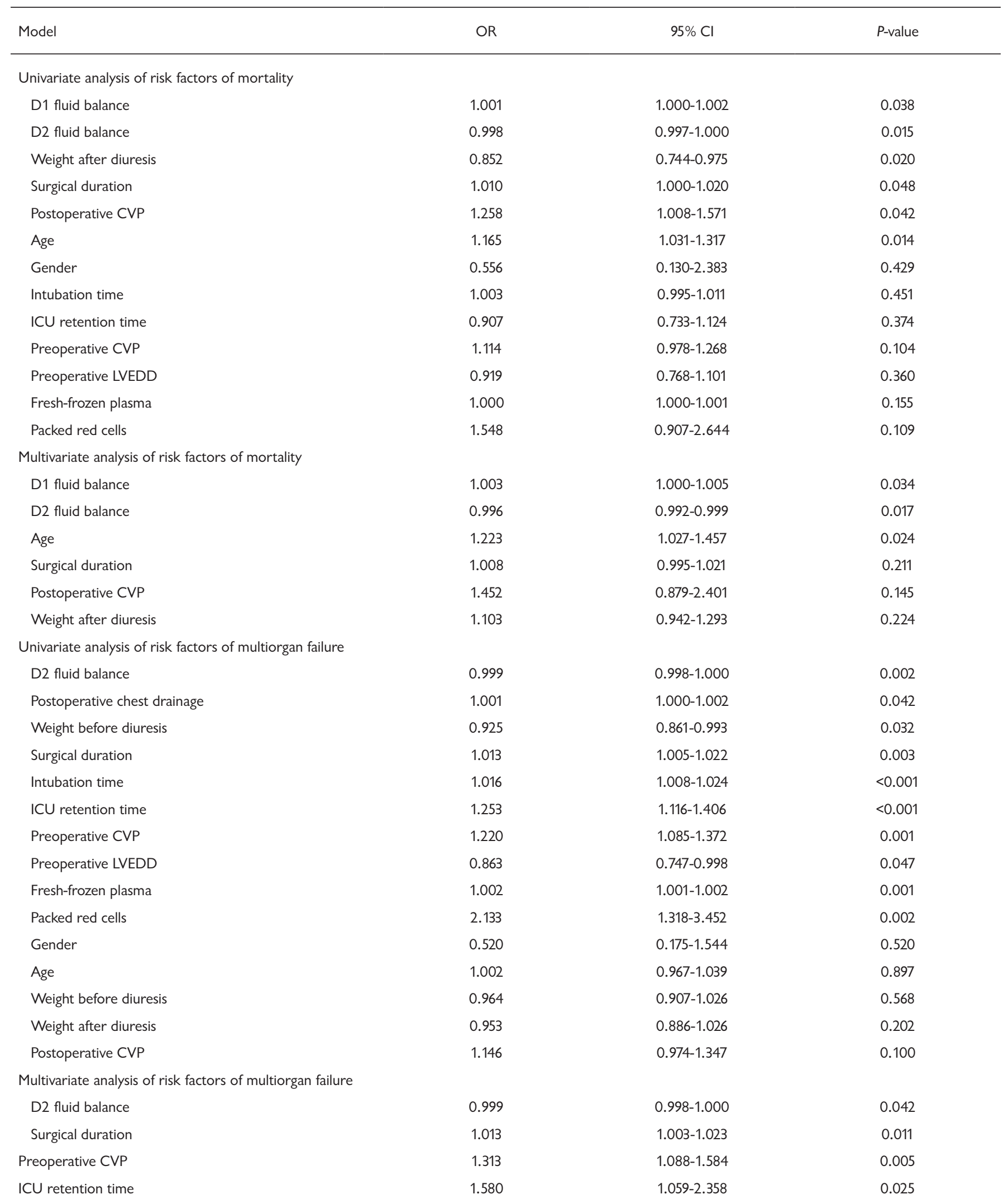




\begin{tabular}{llll} 
Postoperative chest drainage & 1.000 & $0.999-1.001$ & 0.796 \\
Weight before diuresis & 0.943 & $0.867-1.027$ & 0.178 \\
Fresh-frozen plasma & 1.001 & $0.999-1.002$ & 0.333 \\
Packed red cells & 1.768 & $0.777-4.022$ & 0.174 \\
Intubation time & 0.998 & $0.982-1.013$ & 0.169 \\
Preoperative LVEDD & 0.926 & $0.792-1.083$ & 0.335 \\
\hline
\end{tabular}

D0 fluid balance, fluid balance on operation day; D1 fluid balance, fluid balance of the first day following operation; D2 fluid balance, fluid balance of the second day following operation

Table 7. Comparison of patients with tubercular and nonspecific inflammatory pericarditis

\begin{tabular}{|c|c|c|c|}
\hline Male, n (\%) & $14(56 \%)$ & $45(67.2 \%)$ & 0.321 \\
\hline Weight before diuresis, $\mathrm{kg}$ & $56.5 \pm 1.2$ & $55.3 \pm 1.6$ & 0.528 \\
\hline Weight after diuresis, kg & $53.7 \pm 1.0$ & $53.8 \pm 1.3$ & 0.960 \\
\hline Postoperative CVP, $\mathrm{mmHg}$ & $12.4 \pm 0.5$ & $11.6 \pm 0.5$ & 0.260 \\
\hline Preoperative LVEDD, mm & $39.8 \pm 0.5$ & $40.8 \pm 0.7$ & 0.245 \\
\hline Preoperative LVEF, \% & $61.7 \pm 0.8$ & $64.2 \pm 1.0$ & 0.056 \\
\hline Intubation time & $78.2 \pm 12.1$ & $68.4 \pm 10.4$ & 0.553 \\
\hline ICU retention time & $5.5 \pm 0.7$ & $6.5 \pm 1.1$ & 0.443 \\
\hline Surgical duration & $197.6 \pm 9.8$ & $181.3 \pm 8.4$ & 0.223 \\
\hline D0 fluid balance & $-1398.5 \pm 151.0$ & $-1027.0 \pm 93.0$ & 0.055 \\
\hline D1 fluid balance & $-768.8 \pm 156.3$ & $-249.0 \pm 152.2$ & 0.022 \\
\hline D2 fluid balance & $-663.2 \pm 105.8$ & $-657.0 \pm 116.5$ & 0.969 \\
\hline
\end{tabular}

Table 8. Prevalence of causal factors for constrictive pericarditis in treatment centers in the United States, Spain, Iran, and China

\begin{tabular}{lcccc}
\hline Cause & Cleveland Clinic (\%) & Barcelona (\%) & Iran (\%) & Guangxi, China (\%) \\
\hline Idiopathic disease & 46 & 46.7 & 60 & 34.8 \\
Tuberculosis & 3.7 & 6.7 & 22.2 & 4.4 \\
Post-cardiotomy & 37 & 6.7 & 0 & 0 \\
Radiation therapy & 9 & 13.3 & 4.4 & 0 \\
Malignancy & 0 & 26.7 & 8.9 & 0 \\
End-stage renal disease & 0 & 0 & 2.2 & 0
\end{tabular}


acute heart failure and pulmonary edema. Blood transfusion and plasma or albumin should be appropriately applied for patients with anemia or low serum protein [Biçer 2015; Nozohoor 2018].

This low cardiac output state after pericardiectomy might have been due to incomplete pericardiectomy. Nonetheless, postoperative transient interstitial edema could have had an important effect. Correct and effective perioperative management is of great significance to reduce the operative mortality. After pericardiectomy, the heart is in a high-volume load state in a short period of time. At this time, usage of a large amount of diuretics becomes feasible and necessary, which is also one of the key points for further improvement of cardiac function after operation [Welch 2018; Miranda 2017; Hemmati 2017].

Univariate analysis of potential risk factors of multiorgan failure showed that several factors were associated with multiorgan failure, including D2 fluid balance $(P=0.002)$, postoperative chest drainage $(P=0.042)$, weight before diuresis $(P=0.032)$, surgical duration $(P=0.003)$, intubation time $(P<0.001)$, ICU retention time $(P<0.001)$, preoperative CVP $(P=0.001)$, preoperative LVEDD $(P=0.047)$, freshfrozen plasma $(P=0.001)$, and packed red cells $(P=0.002)$. When they were included in multivariate analysis models, multivariate analyses also showed that D2 fluid balance $(P$ $=0.042)$, surgical duration $(P=0.011)$, preoperative CVP $(P$ $=0.005)$, and ICU retention time $(P=0.025)$ are associated with multiorgan failure (Table 6). Figure 5 shows how the postoperative chest drainage affects the incidence of multiorgan failures. (Figure 5)

Pathological discussion: In this series from Guangxi, China, characteristic histopathologic features of tuberculosis of pericardium $(60 / 92,65.2 \%)$ were the most common histopathologic findings, following by the chronic nonspecific inflammatory changes $(32 / 92,34.8 \%$ ). (Table 8 )

Constrictive pericarditis exhibits a heterogeneous pattern and arises from different causes, depending on the geographic area from which it is reported. Thirty-eight percent to $83 \%$ of the cases of constrictive pericarditis are still caused by tuberculosis in developing countries. Table 6 shows the prevalence of causal factors for constrictive pericarditis in treatment centers in the United States, Spain, Iran, and China [Biçer 2015; Nozohoor 2018].

Patient selection and timing of surgery: Pericardiectomy is indicated once the diagnosis of constrictive pericarditis is made. Systematic antituberculosis drugs should be given to patients with constrictive pericarditis caused by tuberculous bacteria. Surgery should be performed after body temperature, erythrocyte sedimentation rate, and general nutritional status are normal or relatively stable and before cardiogenic cachexia and severe live function injury occur.

Study limitations: Limitations of the present study include its retrospective design. There may be a selection bias because of the retrospective nature of the study. Therefore, our findings may have shortcomings and may not be conclusive.

\section{CONCLUSION}

Tuberculosis is the most common cause of constrictive pericarditis in Guangxi, China. Fluid balance of the second day following operation is associated with early mortality and multiorgan failure after pericardiectomy for constrictive pericarditis in our study.

\section{ACKNOWLEDGEMENTS}

This work was supported by Natural Science Foundation of China (grant code: 81360014), Natural Science Foundation of Guangxi (grant code: 2014GXNSFAA118234), Guangxi Key Scientific and Technological Project (grant code: 2013BC26236), and Projects in Guangxi Health Department (grant code: GZPT13-27), and the Projects in Guangxi Health Department (grant code: S2018075).

The authors would like to thank Dr. Li, Department of Cardiothoracic Surgery, The People's Hospital of Guangxi Zhuang Autonomous Region, for his helpful comments.

\section{REFERENCES}

Acharya A, Koirala R, Rajbhandari N, et al. 2018. Anterior Pericardiectomy for Postinfective Constrictive Pericarditis: Intermediate-Term Outcomes. Ann Thorac Surg. 106(4):1178-1181.

Ahmad H, Porter R, Yusuff H, et al. 2019. Extracorporeal membrane oxygenation for right ventricular failure following pericardiectomy. Eur J Cardiothorac Surg. 56(3):625-626.

Armstrong JL, Wojnarski CM, Choi AY, et al. 2020. Constrictive Pericarditis After Lung Transplantation. Transplantation. 104(5):1081-1084.

Bernal W, Wendon J. 2013. Acute Liver Failure. N Engl J Med. 369:2525-34.

Biçer M, Özdemir B, Kan İ, et al. 2015. Long-term outcomes of pericardiectomy for constrictive pericarditis. J Cardiothorac Surg. 10:177.

Calderon-Rojas R, Greason KL, King KS, et al. 2020. Tricuspid Valve Regurgitation in Patients Undergoing Pericardiectomy for Constrictive Pericarditis. Semin Thorac Cardiovasc Surg. S1043-0679 (20)30052-6.

Chandler HK, Kirsch R. 2016. Management of the Low Cardiac Output Syndrome Following Surgery for Congenital Heart Disease. Curr Cardiol Rev. 12(2):107-11.

Chang SA, Oh JK. Constrictive Pericarditis: A Medical or Surgical Disease? J Cardiovasc Imaging. 2019;27(3):178-186.

Depboylu BC, Mootoosamy P, Vistarini N, et al. 2017. Surgical Treatment of Constrictive Pericarditis. Tex Heart Inst J. 44(2):101-106.

Durham RM, Moran JJ, Mazuski JE, et al. 2003. "Multiple Organ Failure in Trauma Patients," Journal of Trauma. 55(4):608-616.

Epting CL, McBride ME, Wald EL, et al. 2016. Pathophysiology of Post-Operative Low Cardiac Output Syndrome. Curr Vasc Pharmacol. 14(1):14-23

Fang L, Yu G, Huang J, et al. 2020. Predictors of postoperative complication and prolonged intensive care unit stay after complete pericardiectomy in tuberculous constrictive pericarditis. J Cardiothorac Surg. 15(1): 148 . 
Gatti G, Fiore A, Ternacle J, et al. 2020. Pericardiectomy for constrictive pericarditis: a risk factor analysis for early and late failure. Heart Vessels. 35(1):92-103.

Hemmati P, Greason KL, Schaff HV. 2017. Contemporary Techniques of Pericardiectomy for Pericardial Disease. Cardiol Clin. 35(4):559-566.

Howitt SH, Grant SW, Caiado C, et al. 2018. The KDIGO acute kidney injury guidelines for cardiac surgery patients in critical care: a validation study. BMC Nephrol. 19(1):149.

Jacob KA, Leaf DE. 2019. Prevention of Cardiac Surgery-Associated Acute Kidney Injury: A Review of Current Strategies. Anesthesiol Clin. 37(4):729-749.

Melo DTP, Nerbass FB, Sayegh ALC, et al. 2019. Impact of pericardiectomy on exercise capacity and sleep of patients with chronic constrictive pericarditis. PLoS One. 14(10):e0223838.

Miranda WR, Oh JK. 2017. Constrictive Pericarditis: A Practical Clinical Approach. Prog Cardiovasc Dis. 59(4):369-379.

Mori M, Mullan CW, Bin Mahmood SU, et al. 2019. US National Trends in the Management and Outcomes of Constrictive Pericarditis: 2005-2014. Can J Cardiol. 35(10):1394-1399.

Nashef SA, Roques F, Sharples LD, et al. 2012. EuroSCORE II. Eur J Cardiothorac Surg. 41:734-44.

Nozohoor S, Johansson M, Koul B, et al. 2018. Radical pericardiectomy for chronic constrictive pericarditis. J Card Surg. 33(6):301-307.

Roussos C, Koutsoukou A. 2003. Respiratory failure. Eur Respir J. 22: Suppl. 47, 3s-14s.

Rupprecht L, Putz C, Flörchinger B, et al. 2018. Pericardiectomy for Constrictive Pericarditis: An Institution's 21 Years Experience. Thorac Cardiovasc Surg. 66(8):645-650.

Unai S, Johnston DR. 2019. Radical Pericardiectomy for Pericardial Diseases. Curr Cardiol Rep. 21(2):6.

Vondran M, Rylski B, Berezowski M, et al. 2019. Preemptive Extracorporeal Life Support for Surgical Treatment of Severe Constrictive Pericarditis. Ann Thorac Surg. 108(5):1376-1381.

Wei S, Zhang L, Cui H, et al. 2019. Surgery for Patients with RadiationInduced Constrictive Pericarditis. Heart Surg Forum. 22(6):E466-E469.

Welch TD. 2018. Constrictive pericarditis: diagnosis, management and clinical outcomes. Heart. 104(9):725-731.

Welch TD, Oh JK. 2015. Constrictive pericarditis: old disease, new approaches. Curr Cardiol Rep. 17(4):20.

Welch TD, Oh JK. 2017. Constrictive Pericarditis. Cardiol Clin. 35(4):539-549.

Yunfei L, Tao L, Yongjun Q. 2019. Re-pericardiectomy for recurrent chronic constrictive pericarditis: left anterolateral thoracotomy is a better approach. J Cardiothorac Surg. 14(1):152. 